\title{
ANÁLISIS DE ASPECTOS DE DESEMPEÑO TÉRMICO Y ACÚSTICO DE AULAS DE LA FACULTAD DE ARQUITECTURA Y URBANISMO-UNNE
}

GALLIPOLITI, Virginia A. / JACOBO, Guillermo J / ALÍAS, Herminia / MARTINA, Pablo / CORACE Juan José

angelinag2@arnet.com.arn / gjjacobo@yahoo.com.ar / heralias2001@yahoo.com.ar / pablo@ing.unne.edu.ar / juancorace@ing.unne.edu.ar

Cátedras Instalaciones II y Estructuras II Facultad de Arquitectura y Urbanismo, Facultad de Ingeniería (FI) (UNNE)

Palabras Clave: Confort térmico, simulación, acústica de aulas.

Keywords: Thermal comfort, simulation, acoustics of classrooms.

\section{RESUMEN}

Se realiza un análisis de las condiciones higrotérmicas y acústicas de tres aulas de la Facultad de Arquitectura y Urbanismo de la UNNE para un período del mes de marzo de 2012. Se tiene por fin del primer estudio elaborar un diagnóstico de la situación, para proponer, en estudios posteriores, criterios de optimización tendientes a lograr una reducción del consumo anual de electricidad, sin disminuir las condiciones de habitabilidad de los espacios interiores. Para esta primera etapa se consultaron las Normas IRAM 11601, 11605 , $11625,11630,11507-1$ y $11507-4$ de confort higrotérmico. Para los cálculos de aislación y absorción acústica, se utilizaron las herramientas impartidas en el curso de acústica de la asignatura Instalaciones II de la FAU.

\begin{abstract}
An analysis of the acoustic and of the hygrothermal conditions of three classrooms of the Faculty of Architecture and Urbanism of the UNNE for a period of March 2012 is realized. It aims to prepare a diagnosis of the situation to propose, in subsequent studies, optimization criteria designed to achieve a reduction of annual electricity consumption without reducing the habitability of the interior spaces. For this first stage, the IRAM 11601, 11605, $11625,11630,11507-1$ and 115074 Standards of hygrothermal comfort were consulted. For the calculations of insulation and acoustic absorption, there were in use the tools given in the course of acoustics of the subject Instalaciones II of the FAU.
\end{abstract}




\section{OBJETIVOS}

Plantear algunas conclusiones en cuanto al desempeño higrotérmico de tres aulas representativas de la Facultad de Arquitectura y Urbanismo para un período de verano, y verificar en forma teórica los comportamientos acústicos de estas en lo que se refiere a reverberación y aislación.

\section{INTRODUCCIÓN}

Las estrategias fundamentales del Programa de Ahorro y Eficiencia energética en edificios públicos de la Subsecretaría de Energía Eléctrica de la Nación las constituyen las medidas edilicias, como orientación, morfología, características de la envolvente y ganancias internas, que, en conjunto, con la utilización de tecnologías eficientes, servicios energéticos prestados y comportamiento del usuario constituyen medidas de eficiencia energética.

Por otro lado, observaciones realizadas por los actores de los distintos niveles de la educación permiten aseverar que las aulas tradicionales son ruidosas y que el ruido interfiere con el desenvolvimiento académico de los alumnos y docentes en general. La revisión bibliográfica permite aseverar que las malas condiciones acústicas reducen la inteligibilidad de la palabra en las aulas, afectando la atención y el rendimiento académico de los alumnos. Además, el ruido torna poco confortable el ambiente laboral de los docentes, convirtiéndose en un factor negativo para su trabajo.

Por lo tanto, este trabajo aborda dos aspectos importantes en el desenvolvimiento académico: situación higrotérmica y de confort térmico de los espacios educativos y los factores que afectan la comunicación de la conversación del docente-alumno en las aulas, demostrando que existen grandes diferencias individuales en las características de los receptores, las que afectan significativamente la acústica requerida en el aula para lograr una efectiva comunicación de la palabra.
El material base para esta investigación fueron los datos relevados en oportunidad del desarrollo del Proyecto "Evaluación térmico-energética de las sedes edilicias de las Facultades de Arquitectura y de Ingeniería de la UNNE" (año 2011-2012). Este proyecto está orientado a la evaluación térmica y energética de los edificios de las facultades mencionadas, y a la propuesta de medidas correctoras, desde el diseño tecnológico, tendientes a lograr un uso más eficiente de la energía para climatización en dichos edificios.

El análisis con monitoreo (medición in situ con instrumentos medidores llamados "Termocuplas") y simulación (software) se realizó durante un período de quince días de verano (2012), en la ciudad de Resistencia, Chaco. Los programas simuladores como SIMEDIF son herramientas informáticas que permitieron validar los datos de temperaturas monitoreadas mediante instrumental y analizar el comportamiento frente a diferentes condiciones climáticas (verano- invierno), detectar problemas de falta de confort (sobrecalentamiento o bajas temperaturas) y evaluar las distintas alternativas. También permitieron ajustar los datos medidos, de forma de conocer su comportamiento y estudiar la eficiencia de posibles alternativas de rediseño.

Para los aspectos acústicos, se procedió a realizar cálculos teóricos de aislación acústica y tiempo de reverberación de las seis aulas involucradas en los análisis del proyecto de investigación citado anteriormente: aulas 4, 6 y 11; Talleres 7A (2. ${ }^{\circ}$ piso der.), Taller 8A (2. piso izq.) y Taller 10A (1.er piso izq.).

\section{EL EDIFICIO DE LA FACULTAD DE ARQUITECTURA, UNNE: TECNOLOGIAS CONSTRUCTIVAS}

El edificio de la Facultad de Arquitectura y Urbanismo (FAU) de la UNNE está implantado en el Campus Universitario de Av. Las Heras en el sector centro-sur 


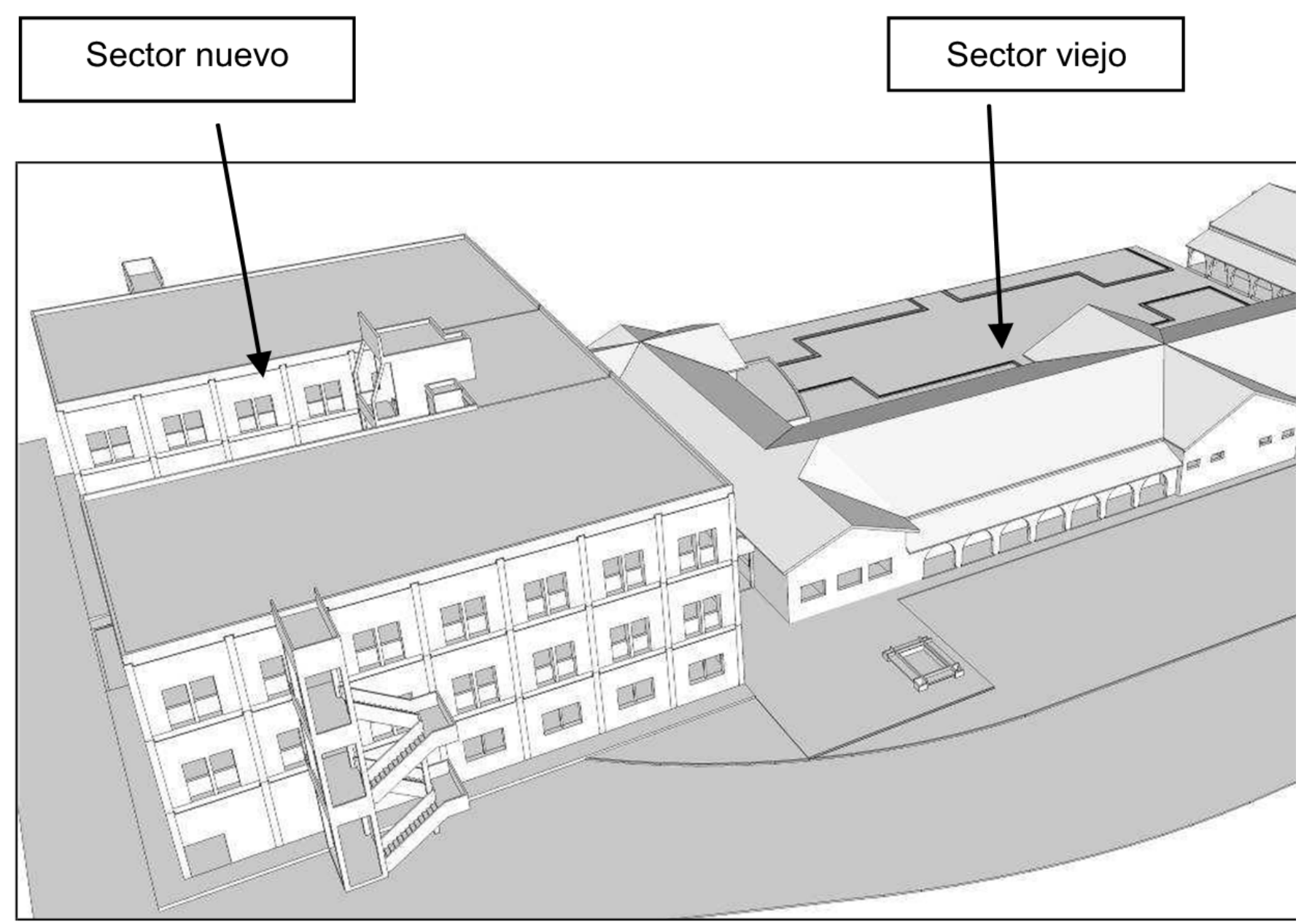

Figura N. 1: Volumetría y sectores denotados como" viejo" y" nuevo" del edificio de la FAU - UNNE

de la ciudad de Resistencia, Chaco (Latitud: 27,45\%; Longitud: 59,05 Oeste; Altitud: $52 \mathrm{msnm})$, en un área urbana de media densidad. El edificio se erigió originalmente a fines de la década de 1950 como un hogar-escuela, y en 1957 se convirtió en la sede universitaria de la UNNE. Presenta dos sectores bien diferenciados en cuanto a su tecnología constructiva:

- El sector "antiguo". Alberga cinco aulas, el sector administrativo, no docente y de maestranza, sector de despachos de autoridades, biblioteca de la facultad, centro de informática, oficinas correspondientes a distintos departamentos y bloques de sanitarios. Constructivamente, se resuelve mediante técnicas artesanales tradicionales, materializándose los muros mediante mampuestos comunes macizos de 0,30 m de espesor, revocados exterior e interiormente, y las cubiertas mediante tejas cerámicas tipo coloniales sobre estructura de madera. A lo largo del tiempo fue adaptado para diversas funciones y actividades interiores, por medio de intervenciones parciales y am- pliaciones, implementadas con diversas tecnologías constructivas. En su interior los espacios se organizan a lo largo de pasillos centrales que funcionan como conectores, por encima de cuyos cielorrasos se desarrollan entrepisos que funcionan como depósitos, constituyendo amplios entretechos.

- El sector "nuevo". Iniciado en la década del 90 y finalizado en 2010, consiste en dos bloques de tres pisos unidos por un bloque principal que funciona como hall y conector. Alberga los espacios interiores de mayores envergaduras en cuanto a volúmenes, cantidades de usuarios e intensidades de uso: seis aulas-taller, un auditorio y núcleos de sanitarios. Se resuelve mediante estructuras portantes prefabricadas de hormigón armado y cerramientos verticales de mampuestos artesanales compuestos (muros dobles con cámara de aire y muros de múltiples capas, para los diferentes niveles). Las carpinterías son de marcos de chapa doblada, sin protección exterior, con vidrios simples tipo float de $4 \mathrm{~mm}$. 


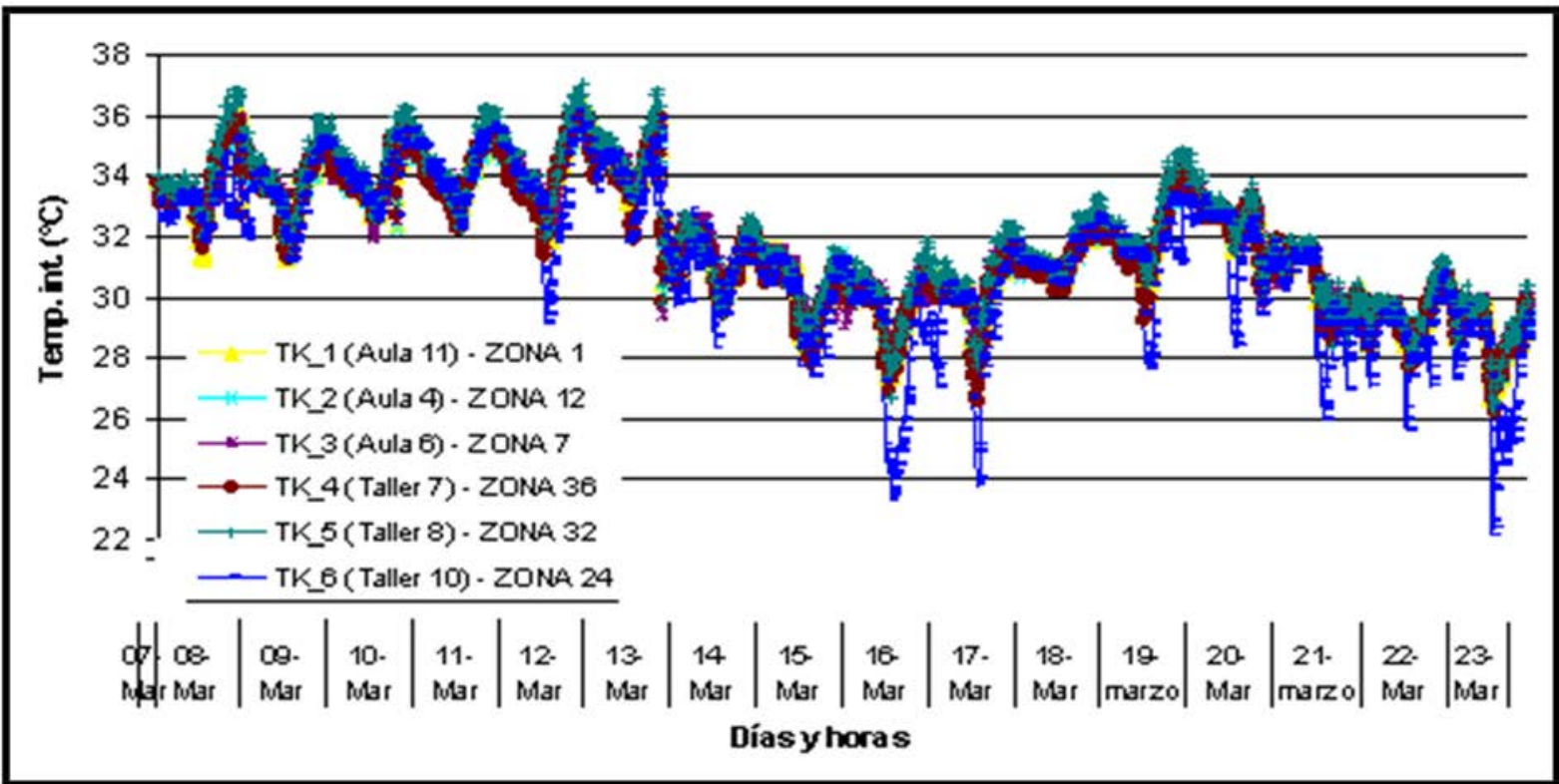

Figura 2 Evolución de temperaturas interiores medidas en seis aulas del edificio de la FAU - UNNE, durante el período del 08/03 al 23/03/2012

\section{MEDICIONES DEL COMPORTAMIENTO TÉRMICO}

Las mediciones térmicas de la muestra definida, durante días con temperaturas representativas de la estación cálida, se realizó en el período comprendido entre el 8 y el 23 de marzo de 2012. Las aulas estuvieron en condiciones de uso normal durante las mediciones, habiéndose relevado tales condiciones de uso (cantidad de usuarios, tipo de actividad, patrón de apertura de aventanamientos y uso de la iluminación artificial, equipamientos y artefactos en uso). De los seis locales, solo las aulas 4 y 6 poseen equipos de climatización electromecánicos, aunque no estuvieron en funcionamiento durante el período de mediciones.

En la figura $N^{\circ} 2$ se muestra, un gráfico que permite visualizar el comportamiento de las temperaturas medidas por los instrumentos durante el período del 8 al 23 de marzo de 2012. Fue construido sobre la base de valores que los sensores de temperaturas iban registrando y almacenando en el Data Loger (base de datos del equipo aportado por la Facultad de Ingeniería). Los datos luego fueron volcados a planillas Excel con las cuales se graficaron temperaturas en función de los días analizados.

De la lectura de los valores de temperaturas interiores medidas de las seis aulas, durante el período de registro, se infiere que entre el 8 y el 13 de marzo, días de los registros térmicos exteriores más altos, las temperaturas de las seis aulas se mantuvieron, durante las 24 horas de cada día, entre los $32^{\circ} \mathrm{C}$ y los $37^{\circ} \mathrm{C}$, esto es, entre 3 y $8^{\circ} \mathrm{C}$ por encima del límite superior de la banda de confort fijada $\left(19^{\circ} \mathrm{C}-29^{\circ} \mathrm{C}\right)$. Tan solo entre el 15 y el 17 de marzo y luego a partir del
21 de marzo, las temperaturas mínimas de los locales descendieron hasta los $26,5^{\circ} \mathrm{C}$ (en horas de mínima exterior, a las 6,00 hs. aprox.), en coincidencia, aunque con retraso de un día, con el brusco descenso de la temperatura exterior iniciado el 13 de marzo. No obstante, durante este lapso, las medias y máximas interiores se mantuvieron en el orden de los $30^{\circ} \mathrm{C}$ a $35^{\circ} \mathrm{C}$ respectivamente, es decir, por encima del límite superior confortable fijado. Las máximas temperaturas interiores se registraron con un retraso de 3 a 4,5 hs. con respecto a las máximas exteriores (que ocurren a las 15,00 hs. aproximadamente). La amplitud térmica exterior diaria registrada fue de $10^{\circ} \mathrm{C}$ para el 8 de marzo (uno de los días más cálidos registrados) y de $5^{\circ} \mathrm{C}$ para el 15 de marzo (día menos cálido registrado); en tanto que la amplitud térmica interior promedio diaria registrada fue de $5^{\circ} \mathrm{C}$.

\section{SIMULACIÓN DE VERANO CON EL PROGRAMA SIMEDIF}

Se realizó una simulación dinámica de desempeño térmico del edificio de la FAU - UNNE con el programa SIMEDIF para el mismo período monitoreado (del 08/03/2012 al 23/03/2012), para las aulas 4, 6 y 11, abarcando representatividad de los dos sectores del edificio (antiguo y nuevo), Se introdujeron los datos de variables ambientales de la ciudad de Resistencia, para el período del 04/03/12 al 25/03/12. En la figura N. ${ }^{\circ} 3$ se ilustran dos pantallas iniciales del Programa Simedif, que permite introducir los datos de características de los locales y otra para ingresar datos del clima. Finalmente, luego de la carga de datos en el programa, arrojó los resultados que se pueden graficar nuevamente en planilla Excel. 


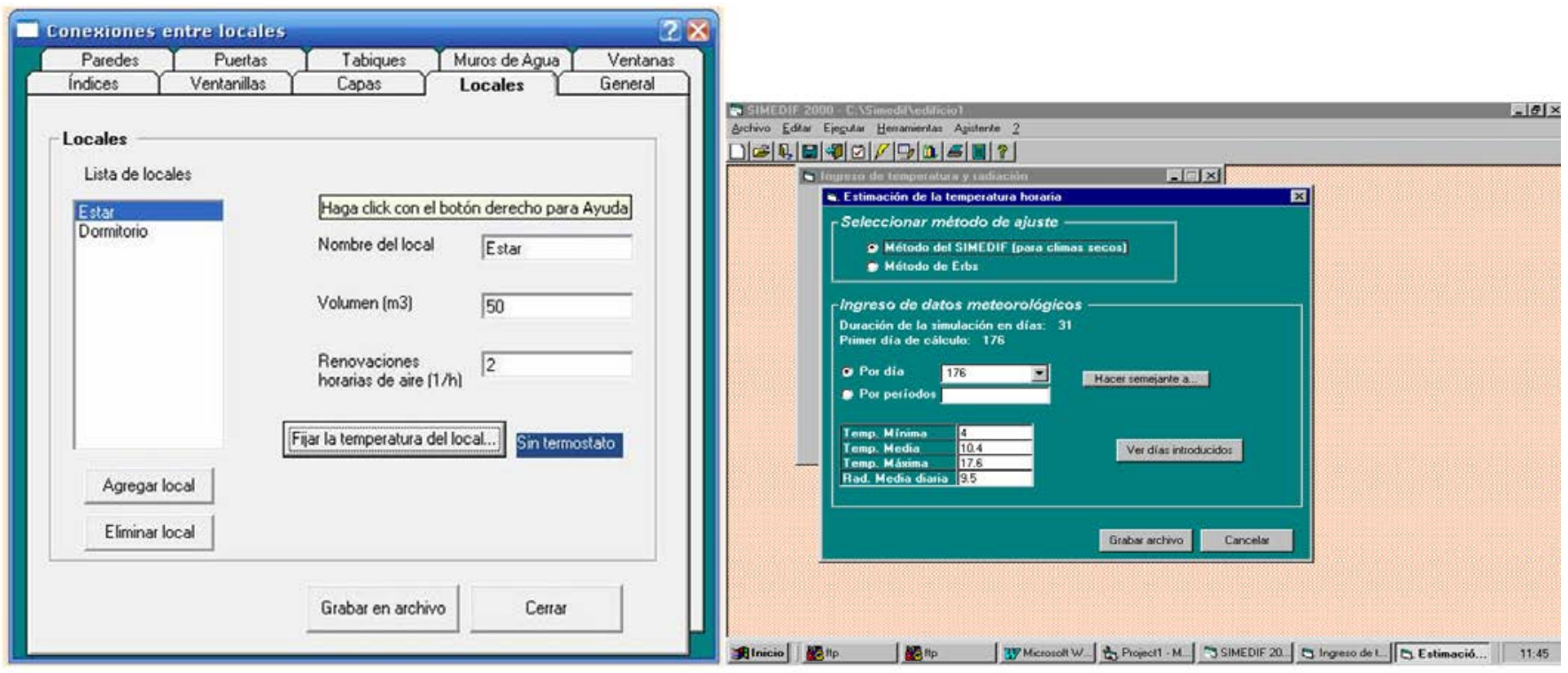

Figura N. ${ }^{\circ}$ : Pantallas del Programa SIMEDIF Cuadro de dialogo "conexión entre locales" y entrada de datos de "Temperatura y Radiación solar"

En la figura $\mathrm{N}^{\circ} 4$ se plasman los resultados arrojados por la simulación con el Programa Simedif para las tres aulas consideradas. También se superponen las gráficas de las temperaturas medidas con los instrumentos.
Las tres aulas de la muestra definida presentaron temperaturas, hasta el día 13/03, que durante las 24 horas de cada día se mantuvieron por encima de la máxima confortable definida para verano $\left(29^{\circ} \mathrm{C}\right)$, re-

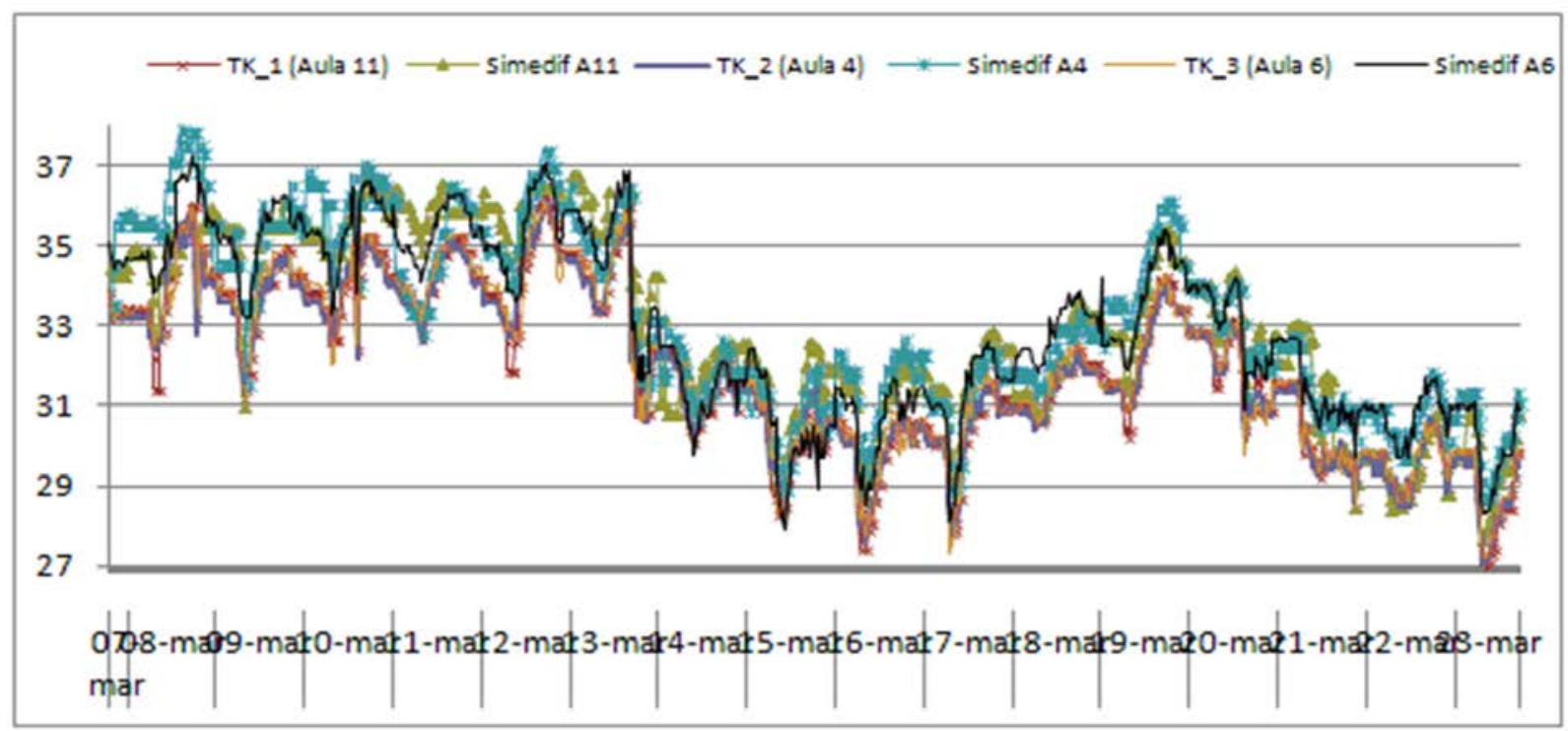

Figura N. ${ }^{\circ}$ 4: evolución de las temperaturas medias simuladas con SIMEDIF y comparadas con las temperaturas medidas o monitoreadas in situ para las aulas 4 , 6 y 11 de la FAU-UNNE

Resultados. Con el modelo físico ingresado a Simedif, según la distribución de locales expuesta, asignados los materiales de cada componente edilicio (muros, techos, pisos, aberturas) y sus respectivos coeficientes de conductividad térmica, densidad y calor específico, ingresadas las cargas internas relevadas y ajustado el archivo de clima, se realizó el análisis térmico para las tres aulas mencionadas. En la fig. 4 se exponen las evoluciones de temperaturas ambiente horarias obtenidas mediante la simulación, para cada aula, durante las 24 hs. de cada día del período del 08/03/2012 al 23/03/2012. Las condiciones mínimas de confort para el análisis térmico en el programa simulador se mantuvieron entre $19^{\circ} \mathrm{C}$ y $29^{\circ} \mathrm{C}$, con 55 a $65 \% \mathrm{HR}$. gistrando mínimas de $33^{\circ} \mathrm{C}$ y máximas de $38,7^{\circ} \mathrm{C}$. A partir del 15/03, las temperaturas internas de las aulas empezaron a registrar máximas de $29-30^{\circ} \mathrm{C}$ y se mantuvieron, la mayor parte de las 24 hs., dentro de la franja prefijada de confort. En la figura 5 se muestra la comparación detallada entre valores de temperaturas medidas y simuladas para el día 12 de marzo. A modo de mejor visualización se pueden comprobar las diferencias entre los resultados del simulador con los datos medidos. Las mayores discrepancias se observan para el aula 11 en períodos en que la temperatura exterior baja, en horas cercanas al mediodía todo el grupo de aulas mantiene un comportamiento bastante uniforme y semejante entre ellas. Se verifica 


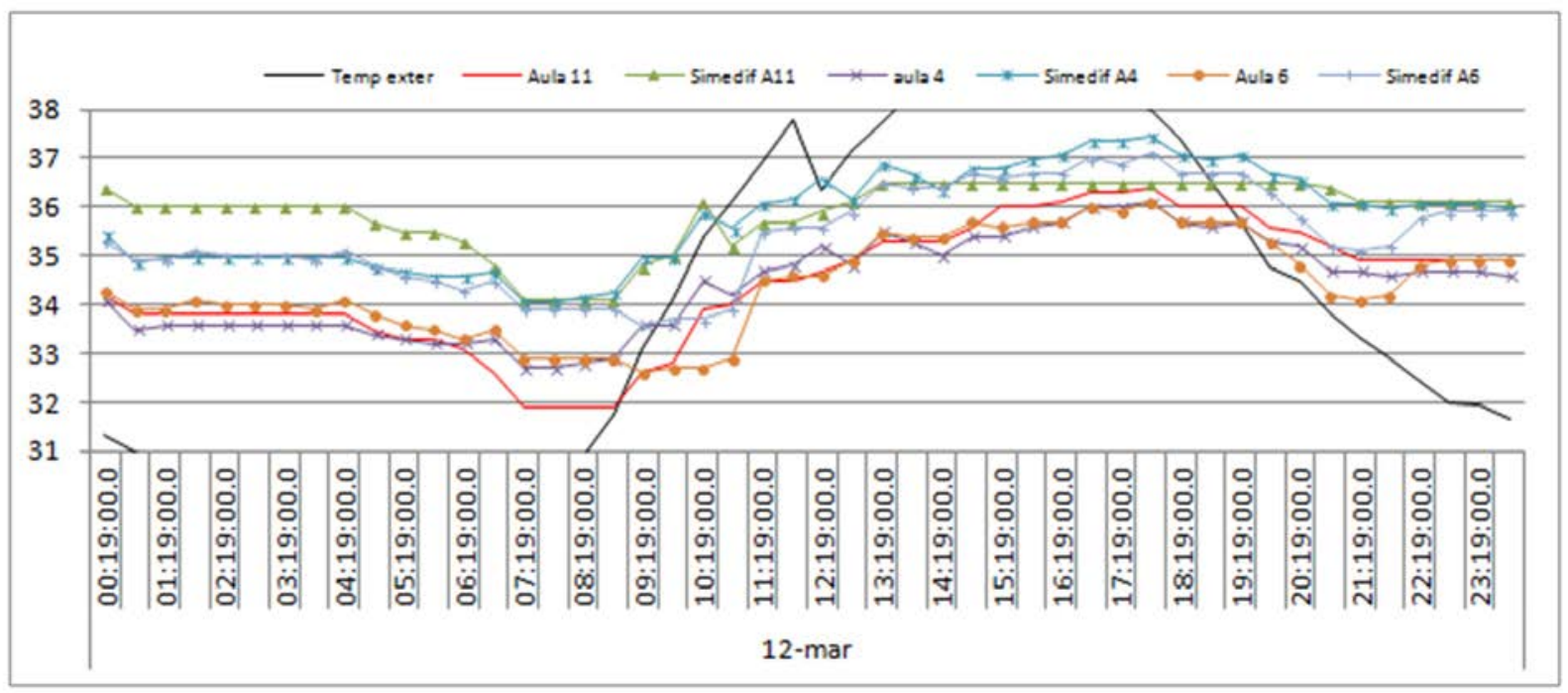

Figura $N^{\circ}$ 5: Resultados y comparación de valores medidos y simulados de temperaturas internas para el día 12 de marzo

una diferencia de $1^{\circ} \mathrm{C}$ a $2^{\circ} \mathrm{C}$ entre valores medidos y simulados.

Se verifica una excelente correspondencia con la temperatura media, aproximadamente $35^{\circ} \mathrm{C}$ y muy buena correspondencia con las temperaturas máximas y mínimas, aproximadamente $1^{\circ} \mathrm{C}$ a $3^{\circ} \mathrm{C}$ de diferencia. En general, coinciden las horas de ocurrencia de temperaturas máximas y mínimas, constatándose en algún caso desfasaje máximo de media hora. Se observa que el aula ubicada hacia el noroeste presenta temperaturas mayores que la del ambiente exterior y amplitudes térmicas más altas que las aulas 4 y 6 , siendo estas las menos cálidas, ya que cuentan con poca ganancia solar directa.

Esto denota que estas condiciones están claramente fuera del área de confort, por lo que es necesario el uso de refrigeración auxiliar convencional durante todo el día.

\section{CORRELACIÓN ENTRE VALORES SIMULADOS Y MONITOREADOS}

La contrastación de los resultados arrojados por la simulación respecto de los resultados medidos se efectuó sobre la base de las dos series de tempera- turas por cada local (medido y simulado), a las que se les aplicó un gráfico de dispersión. A este gráfico se le agregó la línea de tendencia y se obtuvo el coeficiente de correlación (R2) resultante, que indica el porcentaje ajuste de ambas series (figura 6). En general, se obtuvieron aceptables niveles de ajuste para el período simulado y monitoreado: se lograron para las aulas $4(0.886)$ y $11(0.8613)$ y el aula 6 , que no se muestra aquí (0.859), es la que menos correlación presenta, por lo que deberá ajustar el análisis para la optimización necesaria en la modelización física de esta última.

\section{ANÁLISIS DE LAS CONDICIONES ACÚSTICAS DE LAS AULAS DE LA FAU-UNNE}

En general, en los edificios que han sido diseñados sin prestar atención a sus aspectos acústicos, prevalecen superficies reflectoras del sonido. Las escuelas y sedes universitarias argentinas poseen típicas construcciones de ladrillo y revoque duro y liso, pisos de mosaicos, cielos rasos, sillas, bancos y otros mobiliarios duros y altamente reflectores se encuentran dentro de esta tipología.

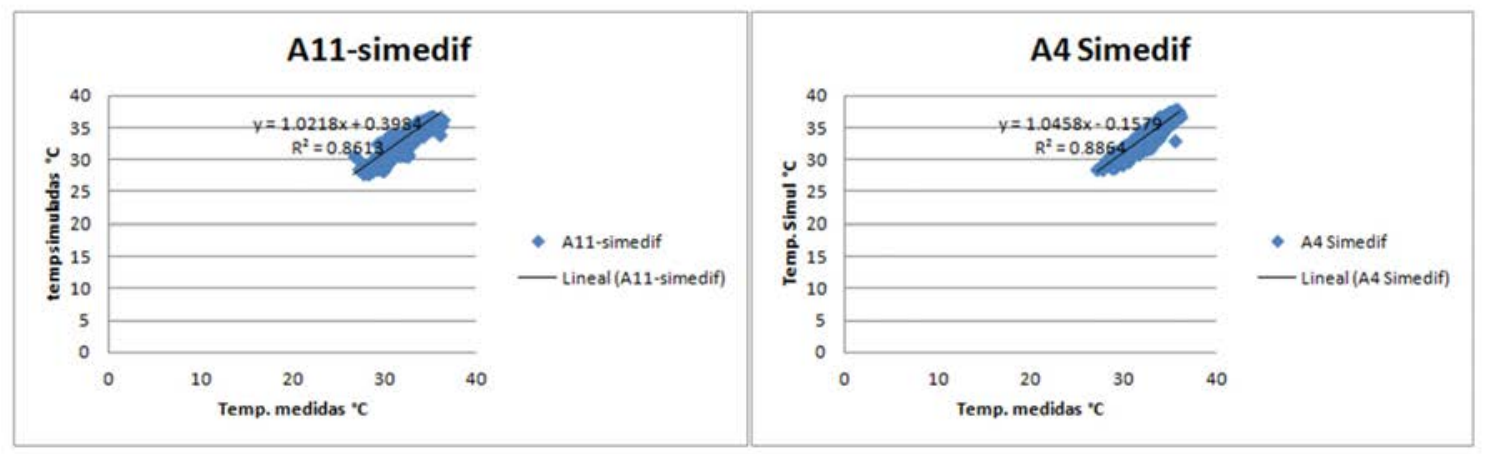

Figura N. ${ }^{\circ} 6$ Correlación entre temperaturas medidas y simuladas, para las aulas 4 y 11 de la muestra definida, durante el período del 08/03 al 23/03/2012 
Entre las posibles causas de un ambiente acústico inapropiado en las instalaciones educativas pueden citarse:

- Mal diseño de las aulas (cuadradas o rectangulares con grandes superficies de paredes paralelas, paredes duras, etc.), lo que conduce a tiempos de reverberación altos.

- Ruido invasivo proveniente de aulas adyacentes y otros ambientes (corredores, patios, etc.); de equipos de aire acondicionado, gimnasios, etc.

- Ruido interno generado por los propios alumnos.

- Ruido invasivo proveniente del exterior: tránsito vehicular, industrias, comercios, etc.

Entre los principales problemas originados por el ruido, deben evaluarse:

- Interferencia en la conversación: cuesta oír la voz del docente. La situación empeora para alumnos con hipoacusias.

- Fatiga en los docentes, que deben alzar su voz por el encima del ruido de fondo para ser oídos, causándoles depresión, angustia, jaquecas, etc.

- Bajo rendimiento académico de los alumnos.

\section{REVISIÓN DE PARÁMETROS Y CRITERIOS INTERVINIENTES}

Las dos condiciones acústicas presentes en la mayoría de los establecimientos educacionales y que impactan negativamente sobre la comprensión del lenguaje hablado son la reverberación y el ruido. La reverberación es la persistencia en el ambiente de energía sonora reflejada, también conocida errónea y vulgarmente como "efecto eco". Es potenciada en espacios con grandes volúmenes y materiales con mínima o nula capacidad de absorción sonora, y su consecuencia es la amplificación del ruido presente.

En efecto, en cualquier espacio cerrado el sonido que se escucha es una combinación del sonido directo (que viaja directamente del emisor hacia el receptor) y el sonido reverberante que viaja desde el emisor y rebota en varias superficies antes de alcanzar el oído del receptor. Técnicamente, es el tiempo en segundos que toma para que un sonido decrezca $60 \mathrm{~dB}$ luego de haber sido generado en la sala. Cuanto más reflectora o reverberante sea esta, mayor será el tiempo que le lleve al sonido decrecer $60 \mathrm{~dB}$. Se denota TR60, o simplemente TR. El segundo factor es el ruido de fondo (RF) o cualquier sonido no deseado proveniente del exterior o interior del edificio que interfiere con la habilidad de las personas para entender el lenguaje hablado. Se lo mide generalmente en dB compensados con la escala $A, d B(A)$.

El presente estudio representa una primera aproximación al análisis más específico, que implica conocimiento de los valores de niveles sonoros presentes en las aulas, sus causas y efectos contraproducentes para el proceso de enseñanza-aprendizaje. En esta instancia se calcularán en forma teórica los principales parámetros acústicos, como el tiempo de reverberación y aislación sonora de las seis aulas tomadas para el análisis higrotérmico anterior. En etapas posteriores se medirán valores con instrumental pertinente y se realizarán encuestas a los diferentes actores del proceso educativo. En este estudio, se tuvieron en cuenta las condiciones arquitectónicas de las aulas (en especial una descripción precisa del tamaño y de las terminaciones de piso, paredes y techo), dado que este importante factor contribuye en ocasiones a los altos niveles de ruido presentes.

\section{CÁLCULO DE VALORES DE TIEMPOS DE REVERBERACIÓN Y AISLACIÓN ACÚSTICA DE DIVISORIOS}

La fórmula de Sabine es utilizada actualmente con muy buena aproximación para hallar valores de tiem- 


\begin{tabular}{|c|c|c|c|c|c|}
\hline \multirow{4}{*}{ AULA } & MATERIAL & SUP. $\left(\mathrm{m}^{2}\right)$ & CANT. & $\begin{array}{c}\text { COEF. } \\
\text { ABSORC. }(\alpha)\end{array}$ & $\begin{array}{c}\text { SUP X COEF } \\
\text { ABSORC. }(\alpha)\end{array}$ \\
\hline \multirow{4}{*}{$\begin{array}{c}\text { Ventanas de vidrio } \\
\text { c/cort } \\
\text { Ventanas de vidrio } \\
\text { s/cort }\end{array}$} & 10,5 & 5 & 0,45 & 23,625 \\
\cline { 2 - 6 } & 10,5 & 2 & 0,027 & 0,567 \\
\cline { 2 - 6 } & Puerta de vidrio & 3 & 1 & 0,027 & 0,081 \\
\cline { 2 - 6 } & Puerta de chapa & 3 & 1 & 0,010 & 0,03 \\
\cline { 2 - 6 } & Pizarra & 14,3 & 1 & 0,170 & 2,431 \\
\cline { 2 - 6 } & Butacas de plástico & 268 & 1 & 0,40 & 107,2 \\
\cline { 2 - 6 } & Alumnos & & $200 / 100$ & 0,45 & $90 / 45$ \\
\cline { 2 - 6 } & Piso granitico & 300 & 1 & 0,010 & 3 \\
\cline { 2 - 6 } & Cielorraso de yeso & 300 & 1 & 0,030 & 5,124 \\
\cline { 2 - 6 } & Paredes & 256,2 & 1 & 0,020 & $238,058 \mathrm{~m}^{2}$ \\
\cline { 2 - 6 }
\end{tabular}

Tabla №1: Plantilla de cálculo para la absorción acústica del aula 11 y posterior cálculo del tiempo de reverberación con fórmulas de Sabine.

po de reverberación de recintos en los que se desea conocer información en cuanto a su acondicionamiento acústico. El físico WALLACE CLEMENT SABINE la desarrolló para calcular el tiempo de reverberación de un recinto en el que el material absorbente está distribuido de forma uniforme. La fórmula relaciona este tiempo con el volumen de la sala, la superficie del recinto y la absorción del sonido total.

Para su aplicación se debe contar con dimensiones de los locales, los elementos dentro de él como cantidad de personas, revestimientos de paredes, pisos y techo, cortinados y todo elemento que pueda aportar reflejo o absorción del sonido emitido dentro. También es importante consultar las planillas de los coeficientes de absorción de los materiales y las gráficas de tiempos óptimos de reverberación para comparar con los resultados obtenidos y proponer una solución.

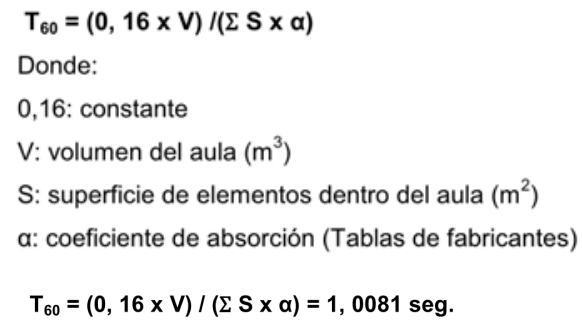

En la Tabla 1, se muestra la planilla de cálculo para el tiempo de reverberación del aula 11. En cantidad de alumnos se colocaron dos valores para obtener coeficientes de absorción diferentes, verificándose el aumento de absorción cuando se ocupa el aula con cien alumnos.

Los valores hallados denotan las falencias en cuanto al acondicionamiento acústico de las aulas analizadas. Lo demuestran las diferencias entre valores hallados de tiempo de reverberación y valores óptimos según recomendaciones de las Normas IRAM 4063, Transmisión de sonido en edificios. Métodos de medición,
1982, IRAM 4043, Aislamiento del sonido en edificios, 1984 e IRAM 4044, protección contra el ruido en edificios. Aislamiento acústico mínimo de Tabiques y muros. 1985. Un TR (tiempo de reverberación) alto corresponde a una absorción total $(\Sigma S \times$ a) pequeña, locales con límites muy reflejantes, y dificulta la diferenciación de sonidos breves emitidos sucesivamente en forma articulada (orador). Las causas de estos ambientes acústicos inapropiados se deben, en gran parte, al mal diseño de las aulas: cuadradas o rectangulares con grandes superficies de paredes paralelas, paredes duras, etc. Los TR deberían reducirse mediante el tratamiento acústico de las aulas con materiales absorbentes.

Los autores C. T. GAGNON y R. HÉTU determinaron que una reducción del TR de 1,6 a 0,6 seg. en los lugares más reverberantes de guarderías de niños resultó en una reducción de $5 \mathrm{~dB}(\mathrm{~A})$ del nivel de exposición al ruido en una sala dada. Además, no se ha encontrado evidencia de que TR muy breves en aulas sean perjudiciales para la propagación de la voz. Al revés, aulas con estas características ayudan a los métodos de enseñanza que utilizan la separación en grupos pequeños que realizan actividades de clase, caso de los Talleres de Arquitectura.

Las soluciones que se pueden proponer desde la asignatura Instalaciones II, y que constituyen los recursos con los que cuenta el alumno que cursa la asignatura, consisten en realizar un nuevo cálculo de superficies necesarias de absorción que deberán ser integradas al local que acondicionar. Para el caso de los talleres, se recalcula el valor de absorción total $(\Sigma S \times$ a) para un tiempo óptimo de 0,85 seg., y se obtiene un valor de $214,58 \mathrm{~m} 2$. Este valor hallado es la cantidad de material absorbente (m2) para colocar en cada aula de taller para reducir el tiempo de reverberación hallado originariamente. Si colocáramos cortinas de tela, su coeficiente sería $a=0,300$. 


\begin{tabular}{|l|c|c|c|c|c|c|}
\hline & Aula 11 & Aula 4 A & Aula 6 A & $\begin{array}{l}\text { Taller } \\
\text { A: } 2^{\circ} \text { piso }\end{array}$ & $\begin{array}{l}\text { Taller 8 8: } \\
\text { A: } 2^{\circ} \text { piso }\end{array}$ & $\begin{array}{l}\text { Taller 10 } \\
\text { A: } 1^{\circ} \text { piso }\end{array}$ \\
\hline $\begin{array}{l}\text { Cant. Máxima de } \\
\text { ocupantes durante el } \\
\text { periodo de monitoreo } \\
\text { (nota: entre las 21hs. y } \\
\text { las 08hs. todas las aulas } \\
\text { estuvieron vacias) }\end{array}$ & 200 & 100 & 50 & 150 & 150 & 150 \\
\hline $\begin{array}{l}\text { Dimensiones en planta } \\
\text { (metros) aproximadas }\end{array}$ & $15 \times 20$ & $6 \times 14.45$ & $6 \times 18$ & $19 \times 35$ & $19 \times 35$ & $19 \times 35$ \\
\hline $\begin{array}{l}\text { Altura (metros) } \\
\text { aproximada }\end{array}$ & 5 & 4.5 & 4.5 & 5 & 5 & 5 \\
\hline To calculados & $1 \mathrm{seg}$ & $1,21 \mathrm{seg}$ & $1,72 \mathrm{seg}$ & $1,11 \mathrm{seg}$ & $1,11 \mathrm{seg}$ & $1,11 \mathrm{seg}$ \\
\hline T60 optimos & $0,80 \mathrm{seg}$ & $0,65 \mathrm{seg}$ & $0,70 \mathrm{seg}$ & $0,85 \mathrm{seg}$ & $0,85 \mathrm{seg}$ & $0,85 \mathrm{seg}$ \\
\hline
\end{tabular}

Tabla N. ${ }^{\circ}$ 2: Tabla resumen de características volumétricas, cantidad de personas, tiempos de reverberación y tiempos óptimos para las seis aulas analizadas de la FAU-UNNE

Este estudio se completará con otras actividades previstas en instancias posteriores que aportarán la información necesaria para definir las soluciones. Se realizarán mediciones y encuestas en aulas, pasillos y sala de lectura de la biblioteca. Por otra parte, resulta de gran utilidad la realización de encuestas entre docentes y alumnos, con el objeto de evaluar la problemática desde el punto de vista subjetivo, de tal manera de establecer correlaciones entre parámetros objetivos (mediciones) y subjetivos (opiniones).

\section{VALORES DE AISLACIÓN ACÚSTICA EN MUROS PERIMETRALES Y TABIQUES INTERIORES}

El aislamiento sonoro de un muro es la resistencia al paso de un sonido de un recinto a otro. Los cambios de presión del aire mueven a los objetos. El impacto de una onda de presión sonora sobre un tabique provoca su vibración. Este movimiento es tan pequeño que normalmente no es visible; sin embargo, es suficiente para provocar sonido que es irradiado al local vecino.

Para hacer vibrar un muro se requiere un esfuerzo, y a mayor masa se requerirá mayor esfuerzo para moverlo y atravesarlo. Parte de ese esfuerzo se disipará en forma de calor, resultando un menor volumen del sonido en el local receptor. Es por ello que los materiales aislantes (muros) deberán tener suficiente masa superficial $(\mathrm{kg} / \mathrm{m} 2)$.

De acuerdo con la Ley de Masa, a mayor masa mayor capacidad de aislación acústica de los elementos. A continuación se verifican los valores de Aislación acústica de los muros perimetrales (al exterior) e interiores de las aulas 4,6 y 11 y talleres 7,8 y 10 .

\section{Aula 6 y Aula 4}

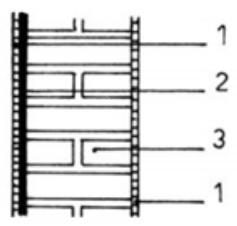

\section{1 revoque grueso reglado MAR}

2 azotado hidrófugo $\mathrm{MCl}$ 1:3

3 ladrillos comunes macizos $\mathrm{e}=30 \mathrm{~cm}$

1 revoque grueso reglado MAR

Taller 10 A Taller 8A y Taller 7A

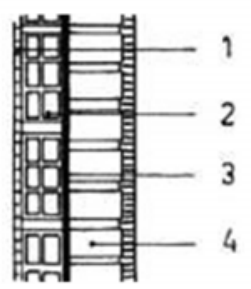

\begin{tabular}{|l|}
\hline 1 revoque grueso reglado \\
MAR \\
\hline 2 ladrillo hueco $8 \times 18 \times 25$ \\
\hline 3 poliestireno expandido \\
\hline 4 ladrillos comunes \\
\hline 1 revoque grueso reglado \\
MAR
\end{tabular}

Tabique interno entre aulas 11 y $12 \mathrm{~A}$

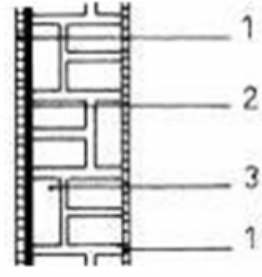

1 revoque grueso reglado MAR 2 azotado hidrófugo $\mathrm{MCl}$ $1: 3$ 3 ladrillos comunes macizos $\mathrm{e}=22 \mathrm{~cm}$

1 revoque grueso reglado MAR 
Aula 11

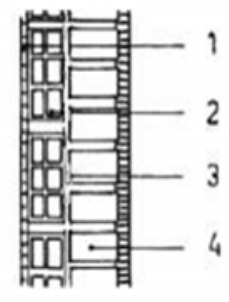

1 revoque grueso reglado MAR

2 ladrillo hueco

3 cámara de aire

4 ladrillos comunes

1 revoque grueso reglado

MAR
Muros aulas 4 y 6: Aislación acústica: 50 DB

Tabique interno entre aula 11 y 12: 47 DB

Muros talleres: $64 \mathrm{DB}$

Muro aula 11: 58,5 DB

Figura N. ${ }^{\circ}$ 7: Tipologías de muros en cerramientos verticales y tabiques internos de aulas de la FAU- UNNE. Características constructivas y cuadro con cálculos de valores de aislación acústica

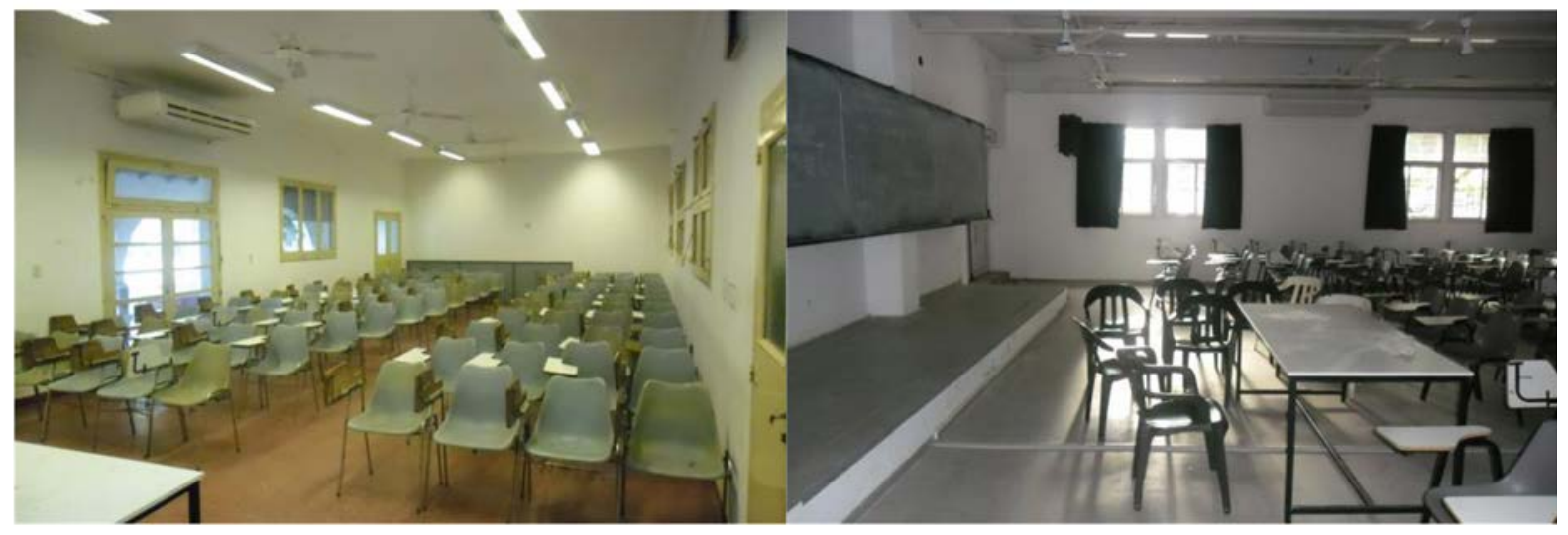

Figura N. 8: Aulas de la FAU- UNNE representativas de los sectores "nuevo" (Aula 11) y "viejo" (Aula 4). Análisis de aspectos acústicos

Vistos estos primeros resultados, se puede realizar un primer análisis señalando que en el sector "nuevo" de la FAU constituido por los talleres, se obtuvieron valores aceptables de aislación acústica de sus cerramientos verticales. Si consideramos un valor de ruido exterior (transito) de 60 DB, los muros aíslan 64 DB, acorde para el trabajo académico. Los muros externos de las aulas 4 y 6 cumplen adecuadamente. El tabique interno entre aulas 11 y 12 cumple satisfactoriamente la aislación acústica entre ellas, considerando un nivel de ruido en cada una de las aulas de 40 DB (conversación normal).

En la figura 8 se aprecian dos de las aulas analizadas, el aula 4 del sector "antiguo" de la FAU y el aula 11 del sector "nuevo".

El aula 11 se ve desfavorecida acústicamente por varios factores. En principio, el valor hallado de aislación acústica de los muros que dan al exterior resulta menor (58,5 DB) que el valor de ruido externo por tránsito vehicular (60 DB). Por encontrarse en la planta baja del edificio de los talleres, los ruidos internos de pasillos y accesos al hall central son permanentes, y solo se aíslan débilmente cuando se cierra la puerta de ingreso al aula, que se realiza, generalmente, en forma intermitente.

\section{CONCLUSIONES}

Por los resultados obtenidos de las mediciones térmicas, contrastados con simulaciones mediante Simedif de tres aulas del edificio de la Facultad de Arquitectura y Urbanismo, el edificio constituye un caso de desempeño térmico deficiente durante días de verano típicos de la zona "lb", con temperaturas interiores por encima del límite superior confortable definido, durante el $95 \%$ del tiempo de mediciones.

Se ha encontrado que el grado de ajuste y aproximación entre los resultados simulados respecto de los medidos resulta razonable (R2 del orden de 0,886 a $0,85)$. De ello se infiere que la modelización utilizada para las simulaciones es válida, aunque susceptible de ser optimizada. Aun así, se considera que es posible generalizar los resultados obtenidos mediante la simulación, incluyendo en el análisis a los locales no monitoreados, con un razonable nivel de confiabilidad.

En función de los resultados obtenidos, se hacen necesarias propuestas de mejoramiento de las envolventes de los locales, que optimicen el desempeño térmico del edificio y que contribuyan a la reducción del consumo eléctrico para climatización artificial. Entre 
dichas mejoras podrían incluirse el aumento de resistencia térmica del conjunto envolvente, así como la provisión de dispositivos de control de incidencia de radiación solar, especialmente en aberturas.

También se exponen los primeros resultados teóricos en cuanto a parámetros acústicos de seis aulas de la Facultad de Arquitectura y Urbanismo. Tres del sector "viejo" y tres del sector "nuevo". Los valores hallados de tiempo de reverberación denotan las falencias en cuanto al acondicionamiento acústico de las aulas. Se calcularon TR (tiempo de reverberación) altos que corresponde a una absorción total pequeña, esto se produce cuando los locales poseen límites muy reflejantes. Las causas de estos ambientes acústicos inapropiados se deben, en gran parte, al mal diseño de las aulas: cuadradas o rectangulares con grandes superficies de paredes paralelas, paredes duras, etc. Los TR deberían reducirse mediante el tratamiento acústico de las aulas con materiales absorbentes.

En cuanto a los valores calculados de aislación acústica de muros y tabiques el sector "nuevo" de la FAU, constituido por los Talleres, presentaron valores aceptables de aislación acústica de sus cerramientos verticales, excepto el aula 11, al que se le agregan pérdidas de aislación por carpinterías aledañas a accesos de pasillos. Los muros del sector nuevo aíslan $64 \mathrm{DB}$, contra un valor de ruido externo de $60 \mathrm{DB}$. Las aulas 4 y 6 cubren cabalmente el valor de aislación de sus muros exteriores, también en sus tabiques internos, aunque las pérdidas por carpintería condicionan estos beneficios iniciales. La colocación de burletes de goma en aberturas seria una primera medida mitigadora. 


\section{BIBLIOGRAFÍA}

Secretaría de energía de la Nación (2001). Programa de ahorro y eficiencia Energética en edificios públicos. Diagnóstico Preliminar de Potenciales de Ahorro Energético. www.energia3.mecon.gov.ar.

Programa SIMEDIF para Windows (2001) FLORES LARSEN, Silvia, LESINO, Graciela, SARAVIA, Luis, D. Alía. INENCO, UNSa, CONICET Salta, Argentina.

ALÍAS H. et ál. (2011) Monitoreo térmico de aulas de la facultad de Arquitectura y urbanismo de la UNNE (Resistencia, Chaco) en días de invierno y condiciones reales de ocupación. (Articulo) Revista AVERMA Volumen 15, pp. 81-89- ISSN 0329-5184, INENCO, Salta.

Profesor Arq. Oscar R. FERREIRA (1985) Apuntes cátedra INSTALACIONES II- FAU-UNNE. Algunos conceptos básicos sobre Acústica en Arquitectura. Editorial FAU.

C. T. Gagnon y R. Hétu. Noise in day-care centers for children, Noise Control Engineering

Journal (30)2, 57-63, 1988. L. Ercoli y A. P. Azzurro (1982.)Grupo Análisis de Sistemas Mecánicos, Facultad Regional Bahía Blanca, Universidad Tecnológica Nacional y A. M. Méndez y J. Stornini CARACTERIZACIÓN SONORA DE AULAS: “Un estudio de los principales parámetros acústicos en aulas argentinas". Laboratorio de Acústica y Luminotecnia, Comisión de Investigaciones Científicas, Pcia. de Bs. Aires. Parque Tecnológico Gonnet, La Plata, ARGENTINA - Norma IRAM 4063, Transmisión de sonido en edificios. Métodos de medición.

Norma IRAM 4043, Aislamiento del sonido en edificios, 1984.

Norma IRAM 4044, protección contra el ruido en edificios. Aislamiento acústico mínimo de tabiques y muros. 1985.

Norma DIN 4109, Noise control in buildings, Sheet 2, Requirements, 1962.

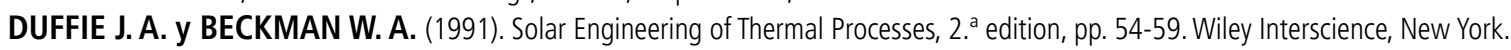

IRAM. Normas de Acondicionamiento Térmico de Edificios: 11605/96: (2007) Condiciones de Habitabilidad en Edificios. Valores máximos de transmitancia térmica en cerramientos opacos. 